\title{
Study of Enterococci with a Special Reference to Species Distribution and Antibiotic Resistance Pattern
}

\author{
Satyendu Saha ${ }^{1 *}$ and Nagaraju Vanaparthi ${ }^{2}$ \\ ${ }^{1}$ NC Medical College, Panipat, Haryana, India \\ ${ }^{2}$ Department of Microbiology, MKCG Medical College, Berhampur, Odisha, India \\ *Corresponding author
}

\section{A B S T R A C T}

\section{Keywords}

Enterococci, Disc diffusion,

Enterococcus faecalis, Species distribution, Vancomycin resistant.

Article Info

Accepted:

17 October 2017

Available Online:

10 December 2017
Glycopeptide-resistant enterococci have become ascendant nosocomial pathogens. Vancomycin resistant enterococci (VRE) can significant increase morbidity and mortality in hospitalized patients. The emergence of VRE is of concern because infections due to these organisms can't be treated with currently available antimicrobial drugs. The present study was conducted with an aim to identify enterococci isolates to the species level and to assess their antibiotic susceptibility profile. Enterococcal isolates were identified as per standard microbiological protocol and antibiotic susceptibility testing was done by Kirby Bauer disc diffusion technique. Majority of isolates were obtained from urine samples. $E$. faecalis followed by E. faecium were the predominant isolates. Vancomycin resistance was noted in a total of $21(10.4 \%)$ enterococcal isolates. The high prevalence of isolation of Enterococcus spp. from clinical specimens and their ability to acquire resistance to the limited number of antibiotics available for treatment of enterococcal infections have highlighted the importance periodic surveillance programmes to identify the colonization with VRE and initiate prompt infection control measures.

\section{Introduction}

During the past 75 years, the "Antibiotic voyage" has met various major and minor turbulences of antibiotic resistance. The abuse of antibiotics like "pill for every ill', selfmedication, over the counter sell, starting broad spectrum antibiotics as the first line of therapy, treating viral fevers with antibiotics, excessive use of antibiotics in agriculture and animal husbandries and other types of misuses have lead to increased resistant rates by many multidrug resistant pathogens called as "Super Bugs" like methicillin resistant Staphylococcus aureus (MRSA), vancomycin resistant Staphylococcus aureus, vancomycin resistant enterococci (VRE), extended spectrum beta lactamase (ESBL) producers. In recent years, enterococci have emerged as important cause of health-care associated or nosocomial infections. In the US, enterococci are second most common cause of hospital associated urinary tract and wound infections and the number three cause of nosocomial blood stream infections (Schaberg et al., 1991). Enterococci demonstrate both intrinsic and acquired resistance to most of commonly used antibiotics. Enterococci are inherently resistant to penicillinase-susceptible penicillin (low level), penicillinase-resistant penicillins, cephalosporins, lincosamides, nalidixic acid, low level of aminoglycoside and low level of 
clindamycin (Murray, 1990). Resistance to penicillin by $\beta$-lactamases, high level aminoglycoside resistance (HLAR), vancomycin, chloramphenicol, erythromycin, high levels of clindamycin, tetracycline, and fluoroquinolone resistance are examples of acquired resistance (Murray, 1990).

Glycopeptide-resistant enterococci have become ascendant nosocomial pathogens. Like MRSA, VRE can significant increase morbidity and mortality in hospitalized patients. The emergence of VRE is of concern because infections due to these organisms can't be treated with currently available antimicrobial drugs (HICPAC, 1995). Therefore present study was conducted with an aim to identify enterococci isolates to the species level and to assess their antibiotic susceptibility profile.

\section{Materials and Methods}

The present study was carried out in the Department of Microbiology of tertiary care hospital for the period of two years (January 2014 to December 2015). The study type was descriptive cross sectional. Enterococcal isolates from various clinical specimens were included in the study. Following were inclusion and exclusion criteria.

Inclusion criteria: Enterococcal isolates from clinical samples like blood, urine, pus, wound swab, catheter tip and other body fluids.

Exclusion criteria: Commensal enterococcal isolates from gastrointestinal tract, female genital tract and oral cavity.

The tests used for identification and speciation of enterococci included colony morphology on blood and MacConkey's agar, morphology on gram staining, catalase test, bile esculin test, growth in the presence of $65 \% \mathrm{NaCl}$, growth at $45^{\circ} \mathrm{C}$ and $65^{\circ} \mathrm{C}$, fermentation of sugars (1\% glucose, mannitol, sorbose, sorbitol, arabinose, raffinose, lactose, and sucrose), arginine hydrolysis, tellurite reduction, $\mathrm{H} 2 \mathrm{~S}$ production and pigment production.

Enterococcal isolates were tested for susceptibility against antibiotics like penicillin, ampicillin, chloramphenicol, erythromycin, amikacin, ciprofloxacin, linezolid, teicoplannin and vancomycin by the Kirby Bauer disc diffusion method. High level aminoglycoside resistance was tested against gentamicin $(120 \mu \mathrm{g})$ and streptomycin $(300 \mu \mathrm{g})$. Antibiotic discs were procured from HiMedia Laboratories Pvt. Ltd. The strength of discs and their zone size interpretative standards were as per the Clinical and Laboratory Standard Institute guidelines (CLSI, 2007). Staphylocococcus aureus ATCC 25923 and Enterococcus faecalis ATCC 29212 were used as control strains.

\section{Results and Discussion}

During the study period, a total of 173 enterococci were isolated from various clinical specimens. As shown in figure 1, majority of isolates were from urine samples (58.9\%) followed by pus (17.9\%) and blood $(10.9 \%)$.

The species wise distribution of enterococcal isolates is shown in figure 2, E. faecalis $(\mathrm{n}=$ $123,71.1 \%)$ followed by E. faecium (22.5\%) were the predominant isolates.

The sample and species wise distribution of enterococcal isolates is shown in table 1. $E$. faecalis was the major isolate from various clinical specimens except for blood. $E$. faecium was the predominant isolate from blood culture.

The antibiotic resistance pattern of Enterococcus spp. is shown in figure 3. 
Among the enterococcal isolates, maximum resistance was noted to penicillin $(91.3 \%)$ followed by erythromycin (77.4\%) and amikacin $(71.1 \%)$. A total of $63(36.4 \%)$ isolates were resistant to high level gentamycin whereas, 65 (37.5\%) isolates showed resistance to high level streptomycin. Vancomycin resistance was noted in a total of $21(10.4 \%)$ enterococcal isolates.

A higher percentage of resistance to all antibiotics tested was noted among $E$. faecium isolates compared to E. faecalis. E. avium, E. durans and E. gallinarum were sensitive to penicillin, ampicillin, high level gentamicin, high level streptomycin, linezolid and vancomycin.

The comparison of antibiotic resistance pattern of E. faecalis and E. faecium is shown in figure 3. All isolates of E. faecium were resistant to penicillin. Although the rate of vancomycin resistance was high among $E$. facecium isolates there was no significant difference observed between vancomycin resistance in E. faecalis and E. faecium isolates (Fischer Exact test, $P$ value 0.167).

In recent years there has been a great deal of concern about the emergence of antimicrobial-resistant organisms. Bacteria and other microbial pathogens have shown a remarkable ability to develop resistance to virtually all class of antibiotics.

The development of vancomycin resistance in Enterococci is perhaps, the most impressive accomplishment of bacteria to date. In contrast to coliforms and other enteric bacteria, the enterococci are rather resilient and can survive for longer period in soil and water, and thus re-enter the food chain.

Table.1 Sample and species wise distribution of enterococcal isolates

\begin{tabular}{|l|l|l|l|l|l|l|}
\hline Clinical specimen & E. faecalis & E. faecium & E. durans & E. gallinarum & E. avium & Total \\
\hline Urine & 78 & 21 & 02 & 01 & - & 102 \\
\hline Pus & 23 & 05 & 01 & - & 02 & 31 \\
\hline Blood & 08 & 09 & - & 01 & 01 & 19 \\
\hline Ascitic fluid & 09 & 03 & - & - & - & 12 \\
\hline Pleural fluid & 03 & 01 & - & - & - & 04 \\
\hline ET tube & - & - & 01 & 01 & 01 & 03 \\
\hline CSF & 02 & - & - & - & - & 02 \\
\hline Total & 123 & 39 & 4 & 3 & 4 & 173 \\
\hline
\end{tabular}

Fig.1 Specimen wise distribution of enterococcal isolates

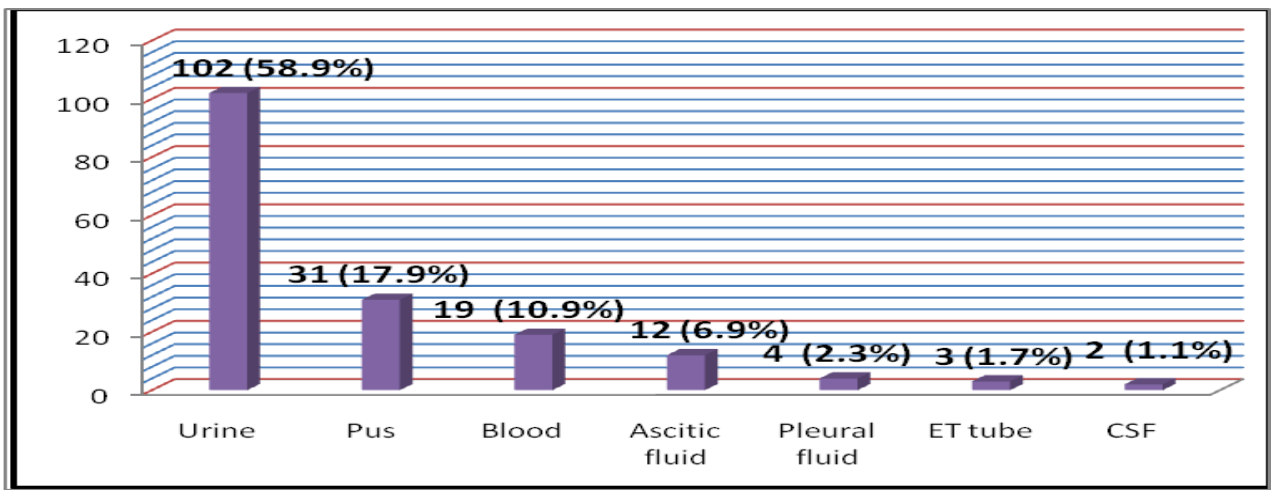


Fig.2 Species wise distribution of enterococcal isolates

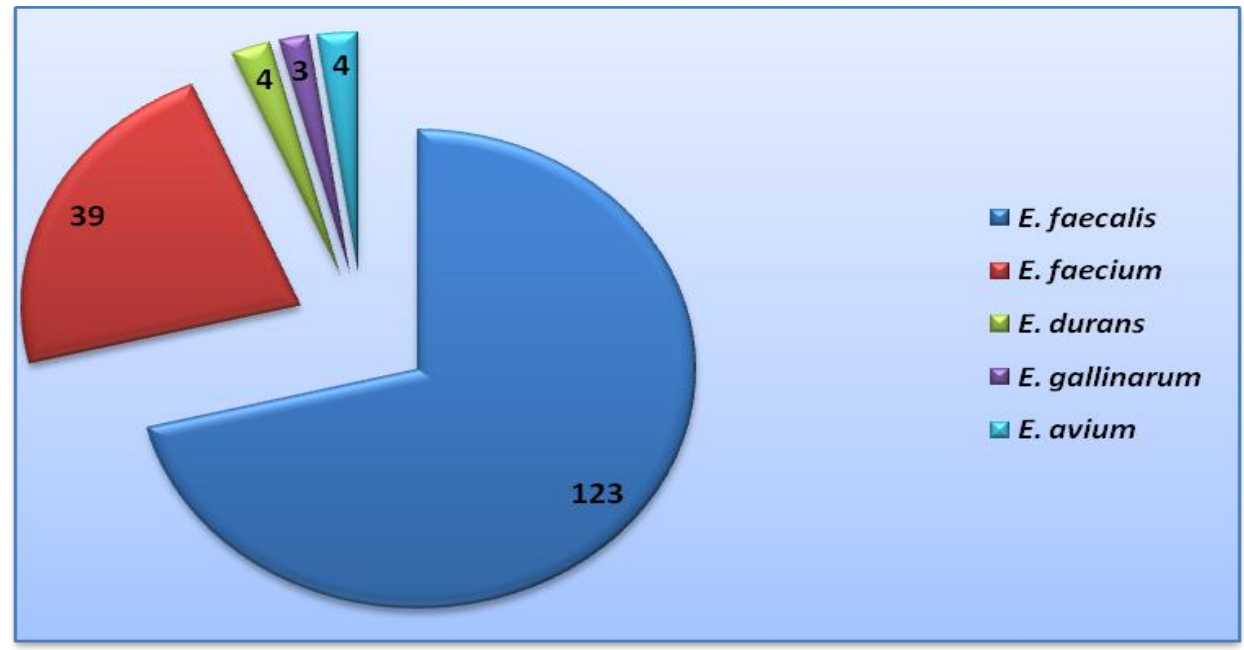

Fig.3 Antibiotic resistance pattern of Enterococcus spp
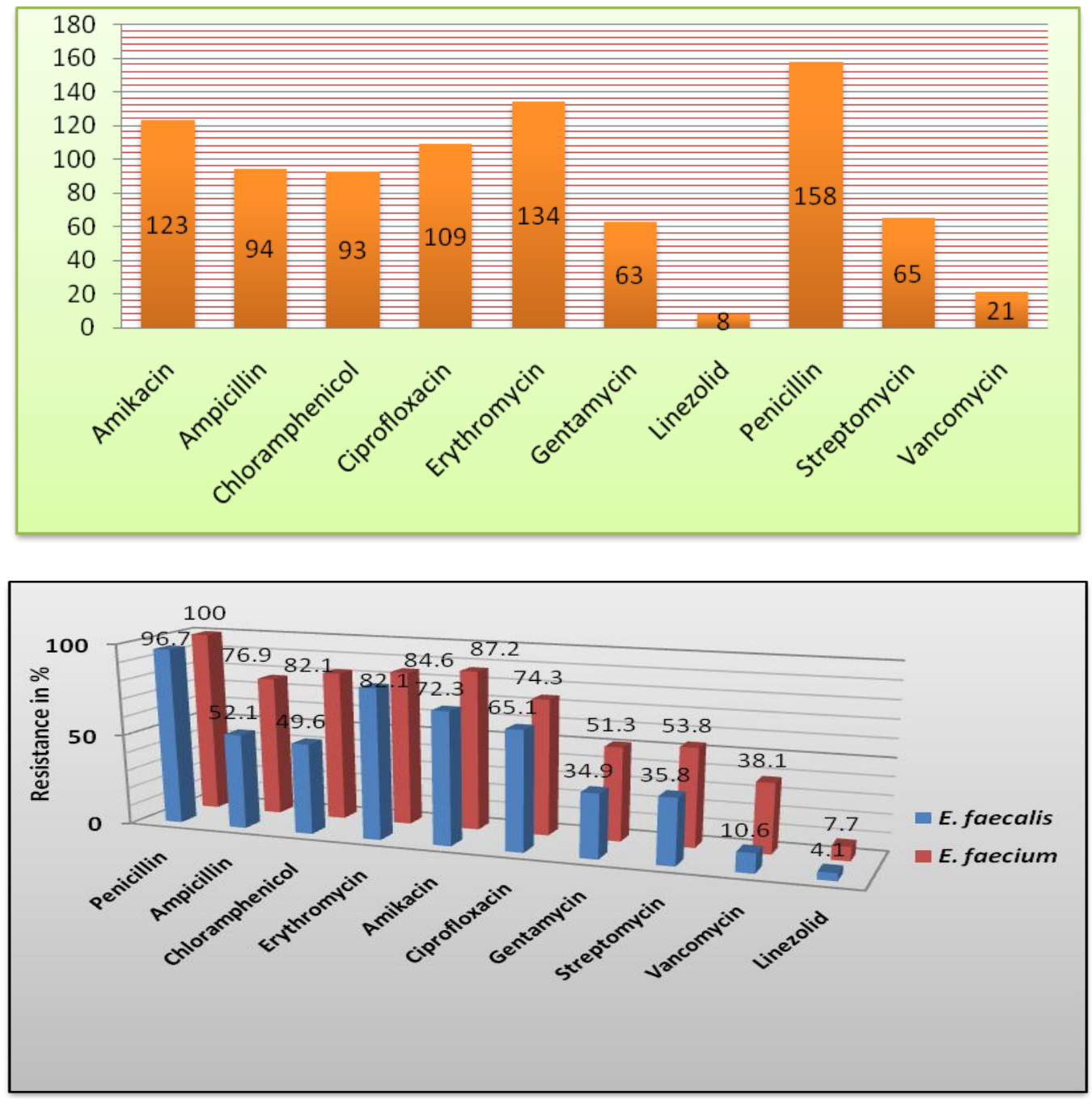
Despite relative lack of pathogenicity, enterococci have emerged as important health-care associated pathogens in many countries. One of the major reasons for increased isolation of Enterococcus spp. from health-care associated infections is their innate resistance to several classes of commonly used antibiotics and, perhaps more important, their ability to acquire resistance to currently available antibiotics, either by mutation or by receipt of foreign genetic material through the transfer of plasmids and transposons (Clevel, 1990). The present study was conducted with an aim to identify the enterococcal isolates to the species level and to assess the antibiotic resistance pattern to widely prescribed antibiotics with a focus on vancomycin resistance.

In the present study, the maximum isolates were obtained from urine followed by pus and blood cultures. Our observation is in accordance to that of Gordon et al., (1992), Udo et al., (2002) and Chaudhary et al., (2007). Nosocomial bacteriuria is the most common type of infection caused by Enterococcus spp (Jett et al., 1994). The bladder, prostate, and kidneys are commonly infected by these bacterial pathogens, especially in patients with structurally abnormal urinary tract or presence of indwelling catheters (Jett et al., 1994). Blood stream infections (BSI) due to Enterococcus spp. are much more common than enterococcal endocarditis (Murray, 1990). Severity of illness, patient's age, and use of broad-spectrum antibiotics are risk factors associated with enterococcal BSI (Green et al., 1995).

Species identification plays an important role in investigation of nosocomial outbreak and selection of most appropriate antimicrobial agent for therapy (Gordon et al., 1992). In severe infections (BSI or meningitis) species identification along with determination of high level aminoglycoside resistance to gentamicin and streptomycin should be strongly encouraged because of variation in susceptibility profile to different classes of antibiotics by different Enterococcus spp (Gordon et al., 1992). In the current study, $E$. faecalis $(71.9 \%)$ and E. faecium (22.5\%) were major species isolated from various clinical specimens. Similar findings were reported by other researchers. E. faecalis is found in higher numbers than E. faecium and other species in the faeces of most healthy adults. E. gallinarum was isolated from a total of 3 $(1.7 \%)$ clinical samples. E. gallinarum is rarely recovered from clinical specimens. However Vandamme et al., (1996) observed that $84 \%$ of the VRE species recovered from faecal specimens of critical patients were $E$. gallinarum.

In the present study, penicillin resistance was observed in $91.3 \%$ of enterococcal isolates. A higher percentage of resistance was noted among E. faecium (100\%) compared to $E$. faecalis $(96.7 \%)$. Similar finding was reported by Karmarkar et al., (2004) and Mendiratta et al., (2008). E. faecalis producing $\beta$-lactamase are resistant to anti-staphylococcal penicillins but are susceptible to ampicillin, amoxicillin, and piperacillin, combined with penicillinase inhibiting drugs like clavulanic acid, sulbactum and tazobactam. E. faecium do not produce penicillinase, yet confer high level resistance (Lin, et al., 1991).

Ampicillin resistance was observed in a total of $94(54.3 \%)$ isolates. Similar rate of ampicillin resistance in enterococcal isolates were reported by many other workers. Unlike other researchers, Karmarkar et al., (2004) reported a higher percentage of ampicillin resistance among E. faecalis. In the present study, ciprofloxacin resistance was observed in $63 \%$ of the enterococcal isolates. Similar observation was reported by Mathur et al., (2003) and Karmarkar et al., (2004). In vitro 
bactericidal effect of ciprofloxacin is highly inoculum dependent; when used alone or in combination with gentamicin in the treatment of experimentally induced endocarditis in rabbits, ciprofloxacin was significantly less effective than penicillin alone or in combination with gentamicin. Therefore ciprofloxacin may not be the best alternative for treating serious enterococcal infections (Gordon et al., 1992).

In the present study, a total of $21(10.4 \%)$ isolates showed resistance to vancomycin. The rate of vancomycin resistance in $E$. faecalis and E. faecium was $10.6 \%$ and $38.1 \%$ respectively. Uttley and colleagues (1988) were the first to report the isolation of vancomycin resistant E. faecium and $E$. faecalis. Vancomycin resistance in enterococcal isolates has been observed to generally be on the rise over the years. The emergence of vancomycin resistance in enterococci poses a serious challenge for physicians treating patients with infections due to these organisms.

In recent years, Enterococcus spp. have been identified as an important cause of a wide variety of infections, particularly UTI and wound infections. These bacterial pathogens have emerged as one of the major causes of health-care associated infections. The rising incidence of enterococcal infection along resistance to commonly prescribed antibiotics has highlighted the importance of adherence to the antibiogram provided by the clinical microbiologist to treating physicians.

\section{References}

Chaudhary, U., Shamma, M., Yadav, A., 2007. Antimicrobial susceptibility pattern of common and unusual Enterococcus species isolated from clinical specimens. J Infect Dis Antimicrob Agents. 24, 55-62.
Clevel, D., 1990. Movable genetic elements and antibiotic resistance in enterococci. Eur J Clin Microbiol Infect Dis. 9, 90102.

Gordon, S., Swenson, J., Hill, B, et al., 1992. Antimicrobial susceptibility patterns of common and unusual species of enterococci causing infections in the United States. J Clin Microbiol. 30, 2373-2378.

Green, M., Schlaes, J., Barbadora, K., Schlaes, D., 1995. Bacteremia due to vancomycin dependent Enterococcus faecium. Clin Infect Dis. 20, 712-714.

Hospital Infection control Practices advisory committee., 1995. Recommendations for preventing the spread of vancomycin resistance: recommendation of hospital Infection control practices advisory committee (HICPAC). Am J Infect Control. 23, 87-94.

Karmarkar, M., Gershom, E., Mehta, P., 2004. Enterococcal infections with special reference to phenotypic characterization and drug resistance. Indian J Med Res. 119, 22-25.

Lin, R., Tan, A., 1991. Enterococcus faecium with high level resistance to gentamicin. Lancet. 338: 260-261.

Mathur, P., Kapil. A., Chandra, R., Sharma, P., Das, B., 2003. Antimicrobial resistance in Enterococcus faecalis at a tertiary care center of northern India. Indian J Med Res. 118:25-8.

Mendiratta, D., Kaur, H., Deotale, V., Thamke., D., Narang, R., Narang, P., 2008. Status of high level aminoglycoside resistant Enterococcus faecium and Enterococcus faecalis in a rural hospital of central India. Indian $\mathbf{J}$ Med Microbiol. 26: 369-71.

Murray, B., 1990. The life and times of the Enterococcus. Clin Microbiol Rev. 3, 46-65. 
Performance Standards for Antimicrobial Testing; Seventeenth Information Supplement. Pennsylvania: Clinical and Laboratory Standards Institute; 2007.

Schaberg, D, Culver, D, Gaynes, R., 1991. Major trends in microbial etiology of nosocomial infection. Am J Med. 91, 72S-75S.

Udo, E., Noura, A., Phillips, O., Chugh, T., 2003. Species prevalence and antibacterial resistance of enterococci isolated in Kuwait hospital. J Med Microbiol. 52, 163-168.

Uttley, A., Collins, C., Naidoo, j., George, R., 1988. Vancomycin-resistant Enterococci. Lancet. I, 57-58.

Vandamme, P., Vercauteren, E., Lammens, C., et al., 1996. Survey of enterococcal susceptibility patterns in Belgium. J Clin Microbiol. 34, 2572-2576.

\section{How to cite this article:}

Satyendu Saha and Nagaraju Vanaparthi. 2017. Study of Enterococci with a Special Reference to Species Distribution and Antibiotic Resistance Pattern. Int.J.Curr.Microbiol.App.Sci. 6(12): 2432-2438. doi: https://doi.org/10.20546/ijcmas.2017.612.281 